\title{
Type Ia Supernovae from Sub-Chandrasekhar Mass White Dwarfs
}

\author{
Stuart A. Sim ${ }^{1}$, Friedrich K. Röpke ${ }^{2,3}$, Markus Kromer ${ }^{3}$, \\ Michael Fink ${ }^{2,3}$, Ashley J. Ruiter ${ }^{3}$, Ivo R. Seitenzahl ${ }^{2,3}$, \\ Rüdiger Pakmor ${ }^{4}$, and Wolfgang Hillebrandt ${ }^{3}$ \\ ${ }^{1}$ Research School of Astronomy and Astrophysics, Mount Stromlo Observatory, Cotter Road, \\ Weston Creek, ACT 2611, Australia \\ ${ }^{2}$ Universität Würzburg, Emil-Fischer-Str. 31, 97074 Würzburg, Germany \\ ${ }^{3}$ Max-Planck-Institut für Astrophysik, Karl-Schwarzschildstr. 1, 85748 Garching, Germany \\ ${ }^{4}$ Heidelberger Institut für Theoretische Studien, Schloss-Wolfsbrunnenweg 35, \\ 69118 Heidelberg, Germany
}

\begin{abstract}
We argue that detonations of sub-Chandrasekhar mass white dwarfs can lead to bright explosions with light curves and spectra similar to those of observed Type Ia supernovae. Given that binary systems containing accreting sub-Chandrasekhar mass white dwarfs should be common, this suggests that a non-negligible fraction of the observed Type Ia supernova rate may arise from sub-Chandrasekhar mass explosions, if they can be ignited. We discuss aspects of how such explosions might be realized in nature and both merits and challenges associated with invoking sub-Chandrasekhar mass explosion models to account for observed Type Ia supernovae.
\end{abstract}

Keywords. hydrodynamics — radiative transfer - methods: numerical - stars: supernovae, white dwarfs

\section{Introduction}

It is widely accepted that Type Ia supernovae (SNe Ia) arise from the thermonuclear explosion of carbon-oxygen (CO) white dwarf (WD) stars. However, the manner in which the explosion occurs and the evolutionary path that leads to it remain topics of very active research. Currently, the best-studied explosion scenarios involve Chandrasekhar mass WDs and it has been shown that explosions of such objects involving a deflagrationto-detonation transition can provide a good match to the observed properties of normal SNe Ia (for recent work, see e.g. Kasen, Röpke, \& Woosley 2009). However, it is disputed whether that model can account for all SNe Ia (see other contributions in this volume) and it is therefore of interest to consider alternative scenarios.

The strongest argument that motivates investigation of sub-Chandrasekhar mass models is the observed rate of SNe Ia. Estimates suggest that the SN Ia rate is roughly one per cent of the rate at which white dwarfs are born (Pritchet, Howell \& Sullivan 2008). This is a challenge for many of the standard explosion scenarios (see other contributions to this volume on Binary Population Synthesis). Consideration of sub-Chandrasekhar mass explosion models potentially opens a wider parameter space of systems that could lead to SNe Ia, making it easier to account for the observed rates (see e.g. discussion by van Kerkwijk, Chang \& Justham 2010).

Here we first summarize results from simple explosion models in which sub-Chandrasekhar mass CO WDs were detonated. We describe results of both nucleosynthesis (Section 2) and radiative transfer (Section 3) calculations for such models and argue that, despite their simplicity, such models can reproduce many of the observed properties of SNe Ia. 
Table 1. Yields from pure detonations of sub-Chandrasekhar mass CO white dwarfs. Specifically, $M_{\mathrm{IME}}$ is the total mass of elements with atomic number in the range $11-20$.

\begin{tabular}{ll|l|l|l|l}
\hline$M_{\mathrm{WD}}\left(\mathrm{M}_{\odot}\right)$ & 0.88 & 0.97 & 1.06 & 1.15 \\
\hline$M_{56} \mathrm{Ni}\left(\mathrm{M}_{\odot}\right)$ & 0.07 & 0.30 & 0.56 & 0.81 \\
$M_{\mathrm{IME}}\left(\mathrm{M}_{\odot}\right)$ & 0.63 & 0.54 & 0.41 & 0.27 \\
\hline
\end{tabular}

In Section 4 we briefly discuss how such explosions might occur in nature. Although subChandrasekhar mass models hold considerable promise, there are also several important constraints that they must address - in particular, the presence of large masses of stable iron-group elements (IGEs) in SNe Ia may present a significant challenge. We comment on this in Section 5 before concluding in Section 6 .

\section{Nucleosynthetic Yields from Detonations of Sub-Chandrasekhar Mass White Dwarfs}

We performed a set of hydrodynamical explosion simulations in which single, hydrostatic sub-Chandrasekhar mass CO WDs were detonated. The detonations were ignited in the center of the WD, leading to one-dimensional explosion models. For each model we then performed nucleosynthesis and radiative transfer post-processing simulations. For details see Sim et al. (2010).

Table 1 lists the ${ }^{56} \mathrm{Ni}$ and intermediate-mass element (IME) yields produced in pure detonation models for a range of initial WD masses $\left(0.88 \mathrm{M}_{\odot}<M_{\mathrm{WD}}<1.15 \mathrm{M}_{\odot}\right)$. Since the densities are higher, a larger fraction of the mass burns to nuclear statistical equilibrium and produces IGEs (predominantly ${ }^{56} \mathrm{Ni}$ ) in the models for more massive WDs. For WDs with mass $M_{\mathrm{WD}} \sim 1 \mathrm{M}_{\odot}$, the expected ${ }^{56} \mathrm{Ni}$-mass from detonation (Table 1) is close to that required to account for the brightness of normal SNe Ia: early phase light curves and nebular spectra consistently suggest that normal SNe Ia typically produce $0.4 \mathrm{M}_{\odot}<M_{56} \mathrm{Ni}<0.8 \mathrm{M}_{\odot}$ (see e.g. Stritzinger et al. 2006).

For very massive (near Chandrasekhar mass) WDs, almost all the mass is expected to burn to IGEs in a detonation and only a very small quantity of IMEs is produced. This low IME yield suggests that detonations of very massive WDs are unlikely to account for the observed properties of normal SNe Ia (see e.g. Arnett, Truran \& Woosley 1971). For lower masses, however, the typical densities are sufficiently low that a significant fraction of the mass is incompletely burned, yielding IMEs. In all cases with $M_{\mathrm{WD}}<1.15 \mathrm{M}_{\odot}$, at least $0.25 \mathrm{M}_{\odot}$ of IMEs are produced - this is sufficient to produce strong spectroscopic features (see below). Also, the models show a clear trend for increasing IME mass with decreasing ${ }^{56} \mathrm{Ni}$-mass. This is similar to the relationship inferred from observations (see e.g. Mazzali et al. 2007).

\section{Synthetic Observables}

The nucleosynthetic yields discussed above suggest that detonation of sub-Chandrasekhar mass CO WDs with $M_{\mathrm{WD}} \sim 1 \mathrm{M}_{\odot}$ can be expected to yield explosions of brightness comparable to SNe Ia that show strong IME features in their spectra. To make a more quantitative assessment, we performed radiative transfer calculations using the ARTIS code (Sim 2007; Kromer \& Sim 2009). Here we present the synthetic spectra and light curves derived from these calculations. 

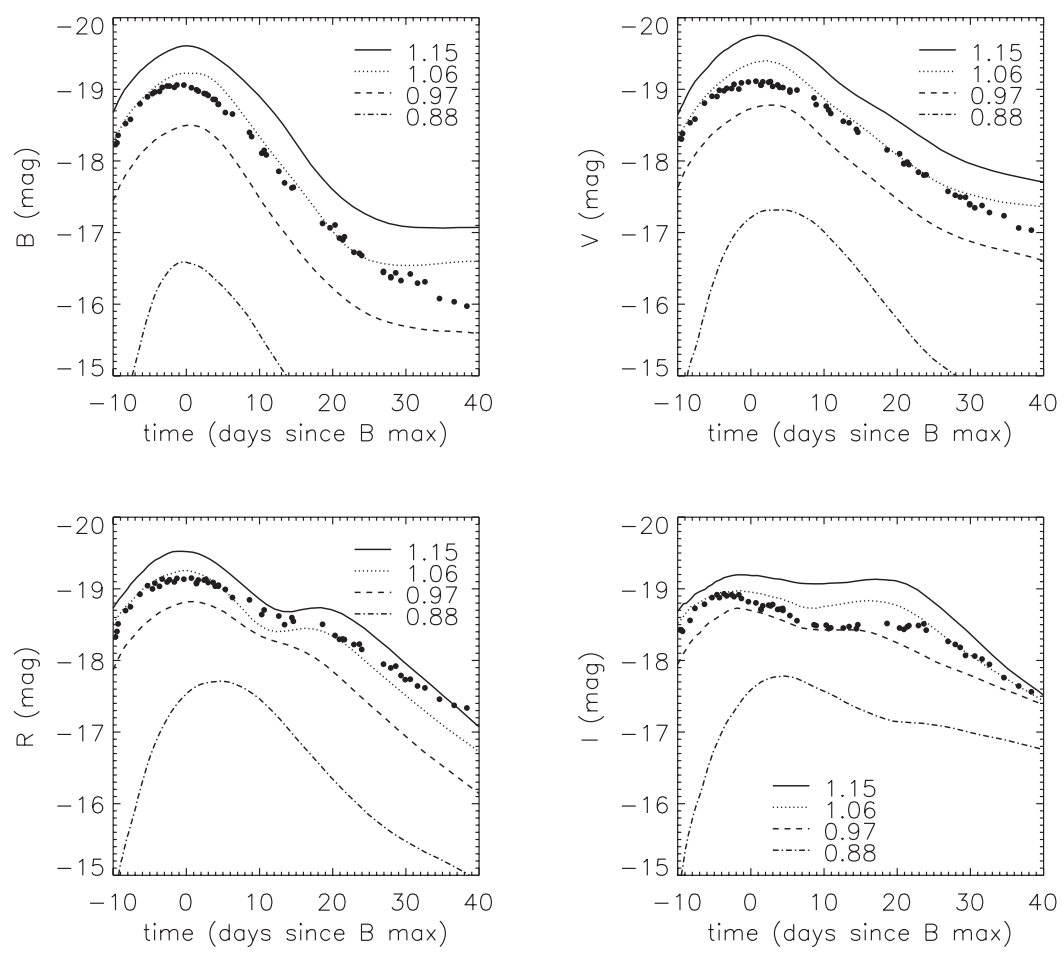

Figure 1. Optical band photometric light curves for detonation of sub-Chandrasekhar mass white dwarfs. Results are shown for four white dwarf masses, ranging from $0.88-1.15 \mathrm{M}_{\odot}$ (continuous curves, labeled in the figure). Observed light curves for SN 2004eo (Pastorello et al. 2007) are over-plotted for comparison (filled circles).

\subsection{Light Curves}

Figure 1 shows synthetic $B-, V$-, $R$ - and $I$-band photometry for our sub-Chandrasekhar mass detonation models with $M_{\mathrm{WD}}=0.88,0.97,1.06$, and $1.15 \mathrm{M}_{\odot}$ (see Sim et al., 2010 for details). For comparison, we have over-plotted observations of SN 2004eo from Pastorello et al. (2007).

Although imperfect, the agreement between the model light curves and observations is remarkably good. The model with $M_{\mathrm{WD}}=0.88 \mathrm{M}_{\odot}$ is clearly too faint and red to account for normal-brightness SNe Ia, but the models with $M_{\mathrm{WD}}$ close to $1 \mathrm{M}_{\odot}$ are a good match in several respects. The light curve rise and decline timescales are in reasonable agreement with observations. Note, in particular, that the light curves do not peak too early, despite the relatively low ejecta masses. The optical colors around maximum light are also a good match to observations. In addition, the brighter models $(0.97,1.06$ and $\left.1.15 \mathrm{M}_{\odot}\right)$ all predict very similar luminosity in the first maximum of the near-infrared light curves (see e.g. the $H$-band light curves shown in Figure 2). This is consistent with the observation that SNe Ia are much better standard candles in the near-infrared than in the optical (see e.g. Krisciunas et al. 2004).

\subsection{Spectra}

Figure 3 shows optical spectra for our sub-Chandrasekhar mass detonation models at the epoch of maximum light in $B$-band. All the models clearly show strong spectral features associated with IMEs, in particular the Si II lines $(\sim 6100 \AA)$ that characterize SNe Ia (S II and $\mathrm{Ca}$ II are also clearly present). The Si line velocities at maximum light are comparable 


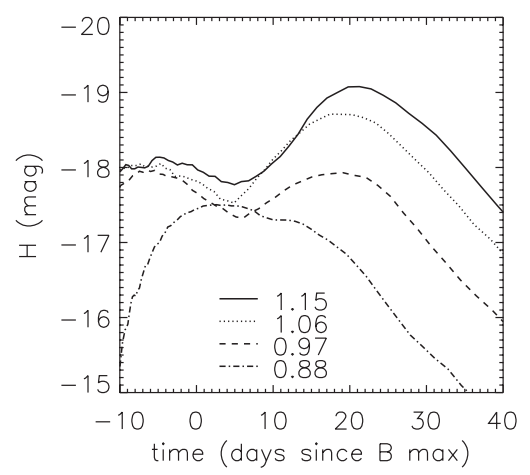

Figure 2. Near-infrared $H$-band light curves for the same models shown in Figure 1.

to those typically observed (see e.g. Benetti et al. 2005), ranging from $\sim 9,000 \mathrm{~km} \mathrm{~s}^{-1}$ in the $M_{\mathrm{WD}}=0.97 \mathrm{M}_{\odot}$ model to $\sim 13,000 \mathrm{~km} \mathrm{~s}^{-1}$ for $M_{\mathrm{WD}}=1.15 \mathrm{M}_{\odot}$. Also, the strength of the bluer Si II $\sim 5800 \AA$ feature relative to the stronger $\sim 6100 \AA$ feature is greater in the less massive (fainter) models. This is also consistent with observational trends (e.g. Nugent et al. 1995).

\section{Triggering a Detonation of a Sub-Chandrasekhar Mass WD}

Taken together, the synthetic light curves and spectra described above show that detonations of CO WDs with mass $M_{\mathrm{WD}} \sim 1 \mathrm{M}_{\odot}$ yield explosions that are as bright as normal SNe Ia and that display the spectroscopic features that are widely used to classify transients as SNe Ia. However, to investigate whether such explosions could be realized in nature it is necessary to consider how a detonation might be triggered. For a successful model, the triggering process should not adversely affect the level of agreement between the synthetic observables and the observations. In addition, if sub-Chandrasekhar mass models are to explain a substantial fraction of the observed SNe Ia population, the triggering mechanism must preferentially operate for $M_{\mathrm{WD}} \sim 1.0 \mathrm{M}_{\odot}$ : for $M_{\mathrm{WD}}<0.9 \mathrm{M}_{\odot}$, detonation would yield only a small ${ }^{56} \mathrm{Ni}$-mass (see Table 1 ), giving rise to an explosion that is less luminous than a typical SNe Ia (but still easily observable). Given that we generally expect low-mass WDs to be more common than high-mass WDs, this implies that either the progenitor evolution or the explosion mechanism leading to a population of sub-Chandrasekhar mass models must be more efficient for systems with $M_{\mathrm{WD}} \sim 1.0 \mathrm{M}_{\odot}$ than e.g. 0.6 $\mathrm{M}_{\odot}$, unless it contributes only a small fraction of total SNe Ia rate.

The best-studied scenario involving the detonation of a sub-Chandrasekhar mass CO WD is the "double detonation" model. This applies to binary star systems in which a primary CO WD accretes He-rich material from a companion star. If a sufficiently massive layer of accreted He builds up, it may detonate. A surface He detonation can trigger a secondary detonation of the underlying CO WD, leading to complete disruption of the star (see e.g. Woosley \& Weaver 1994, Livne \& Arnett 1995, and references therein). This explosion mechanism appears to be robust for a wide range of system parameters provided that detonation of a surface He layer occurs, subsequent detonation of the underlying CO WD is plausible, even for the least-massive He layers that might ignite explosively (Fink et al. 2010). The progenitor systems for this mechanism may be rather common - Ruiter et al. (2011) found a rate for supernovae produced via this channel that is comparable to the $\mathrm{CO}+\mathrm{CO} \mathrm{WD}$ merger rate. This is sufficiently high that this mechanism could contribute significantly to the observed population. 

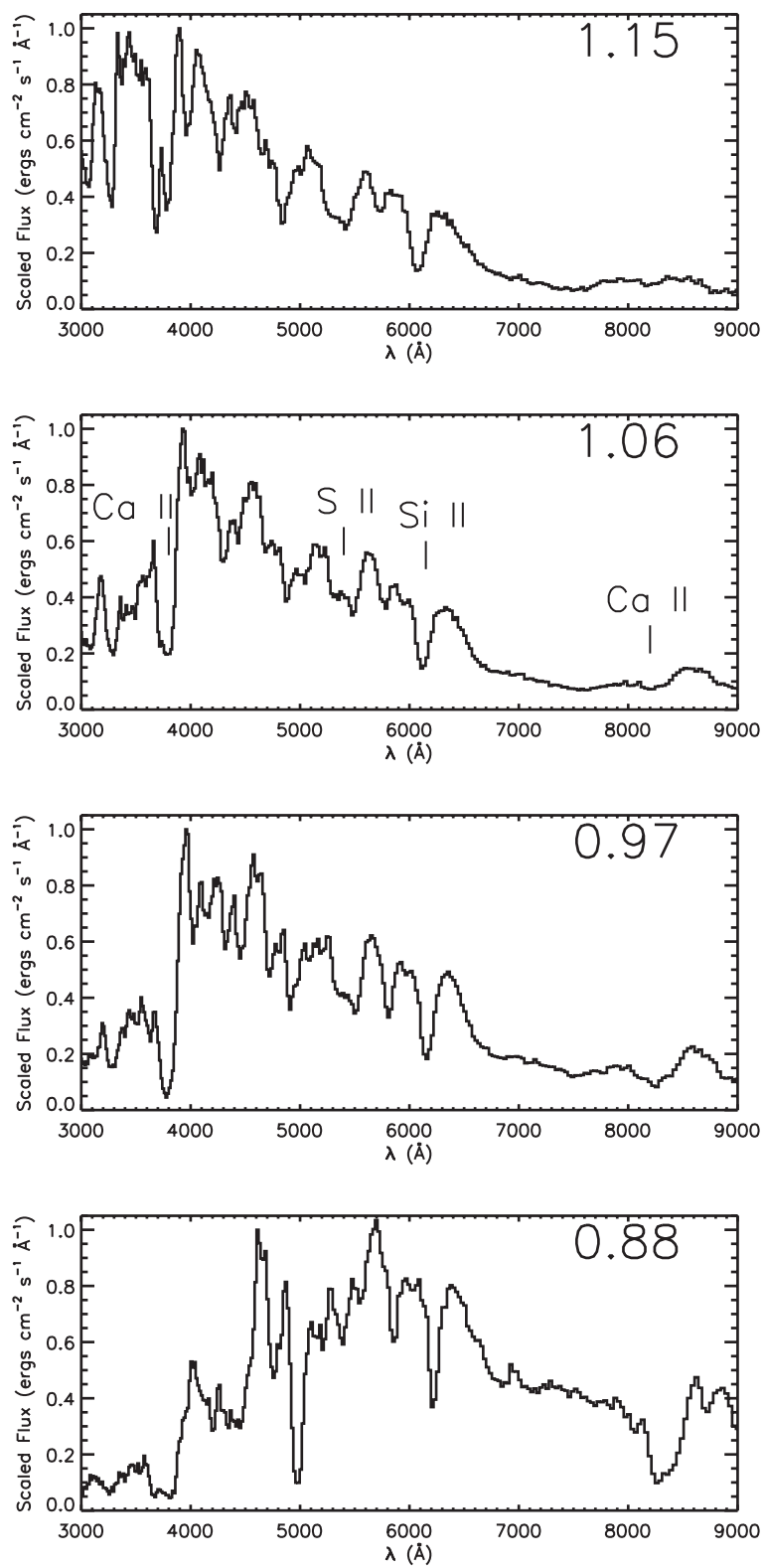

Figure 3. Optical spectra at the epoch of maximum light in $B$-band for the same models as shown in Figure 1 (panels are labeled with the white dwarf mass in $M_{\odot}$ ). The flux is re-scaled to a maximum of 1.0 in each panel. Some of the IME spectral features are labeled in the second panel.

The main drawback for the double-detonation model, however, is that the ejecta from the CO WD are enshrouded by the ashes from burning of the He layer. The He detonation produces a significant amount of IGEs at high velocity. This leads to light curves and spectra that are inconsistent with observations of normal SNe Ia (see e.g. Höflich et al. 1996 and Nugent et al. 1997). Even for the most optimistic models (in which the Helayer mass is minimal), such problems persist (see e.g. Kromer et al. 2010 and Woosley \& Kasen 2011). It is noteworthy, however, that the difficulties in reconciling these models 
with observations primarily stem from the completeness of nuclear burning of the He layer: as discussed by Kromer et al. (2010), slightly less-complete burning of the He layer than predicted in the models could dramatically improve the comparison with observation. This might be achieved by e.g. lower densities or mixing of some heavier elements (e.g. C) into the He prior to explosion. In this context, further investigation of systems with rapid dynamical mass transfer from He WDs to CO WDs is particularly interesting. Simulations have suggested that He detonation might be triggered by knots in the accretion stream (Guillochon et al. 2010) - if this happens when the accreted He is mostly hot and at low-density it could lead to less completely processed nuclear ash, as required. However, it has yet to be shown that He detonation in such systems can lead to a secondary core detonation - to date most focused studies of the secondary CO detonation have assumed a spherically symmetric He layer.

An alternative to the $\mathrm{He}+\mathrm{CO}$ double detonation model is that in which detonation of a CO WD primary occurs as a result of a merger with a CO WD companion. Pakmor et al. (2010) have argued that prompt detonation might occur in such a merger if the mass-ratio is high - they showed that a binary pair of $0.9 M_{\odot}$ WDs might detonate during the merger process and could lead to a sub-luminous 1991bg-like SNe Ia. In their initial study, an equal-mass WD pair was considered but it has been argued that the same type of explosion is plausible for a $0.9 M_{\odot}$ primary if the mass-ratio is greater than $q \sim 0.8$ (Pakmor et al. 2011). If prompt detonation occurs in a merger, there is insufficient time for the density distribution of much of the $\mathrm{CO}$ material to be significantly affected in general the less massive WD will be more disrupted while the primary will largely remain intact. Thus detonation during a merger is likely to yield an explosion that is rather similar to that of a pure detonation model for the more massive WD, regardless of the fact that the total mass of the system might exceed the Chandrasekhar limit. (Of course, the ejecta will also contain material from the secondary - C, O and probably some of their partial burning products - but this will not adversely affect the spectra as strongly as the He-burning products in the double-detonation model.) The expected rate for $\mathrm{CO}+\mathrm{CO}$ mergers that could lead to prompt detonations is uncertain since it depends sensitively on the range of $q$-values for which detonation could be realized, which may itself be a strong function of the primary mass - this is very hard to determine owing to the complexities of simulating the merger inspiral (e.g. Dan et al. 2011) and the formation of detonations (e.g. Seitenzahl et al. 2009). In our view, however, this potential channel is worthy of further investigation as a possible means to account for some fraction of normal brightness SNe Ia.

Recently, van Kerkwijk et al. (2010) discussed the possibility that CO+CO mergers might lead to SNe Ia in systems where the total mass is below the Chandrasekhar mass. Rather than having detonation trigger during a violent merger, they argued that it might occur somewhat later due to compressional heating as the material from the secondary is accreted. This is an attractive idea since sub-Chandrasekhar mass mergers may be common but it remains unclear whether detonation can really be achieved in this case.

\section{Stable Iron Group Elements}

In Section 3, we showed that sub-Chandrasekhar mass explosions can account for the brightness, light curve time scales, colors and spectral features around maximum light. However, late-phase observations of SNe Ia indicate that a significant mass of stable IGEs (mostly ${ }^{54} \mathrm{Fe}$ and ${ }^{58} \mathrm{Ni}$ ) must be produced in the explosion - Mazzali et al. (2007) suggest that all SNe Ia contain $\sim 0.1$ to $0.3 \mathrm{M}_{\odot}$. This is an important constraint that is more restrictive for sub-Chandrasekhar detonation models than more standard scenarios. 
Table 2. Iron group element yields from pure detonations of a sub-Chandrasekhar mass $1.06 \mathrm{M}_{\odot} \mathrm{CO}$ white dwarf for various initial mass fractions of ${ }^{22} \mathrm{Ne}$.

\begin{tabular}{l|c|c|c}
\hline$X\left({ }^{22} \mathrm{Ne}\right)$ & $M_{56}{ }_{\mathrm{Ni}}\left(\mathrm{M}_{\odot}\right)$ & $M_{58} \mathrm{Ni}\left(\mathrm{M}_{\odot}\right)$ & $M_{54} \mathrm{Fe}\left(\mathrm{M}_{\odot}\right)$ \\
\hline 0.0 & 0.55 & $9 \times 10^{-4}$ & $2.6 \times 10^{-5}$ \\
0.025 & 0.51 & 0.021 & 0.026 \\
0.075 & 0.43 & 0.052 & 0.087 \\
\hline
\end{tabular}

In Chandrasekhar-mass delayed detonation models, such a large mass of stable IGEs can be produced during the deflagration phase (see e.g. Seitenzahl, Ciaraldi-Schoolmann \& Röpke 2011 for results of recent three-dimensional simulations). However, in pure detonation sub-Chandrasekhar mass models there is insufficient high-density burning for electron captures to create these stable isotopes. Nevertheless, sub-Chandrasekhar mass detonation models will produce some stable iron group material when the progenitor metallicity is taken into account. For non-zero metallicity, a CO WD is expected to contain a small mass fraction of ${ }^{22} \mathrm{Ne}$. During the explosion, this ${ }^{22} \mathrm{Ne}$ provides a neutron excess which alters the nucleosynthesis and gives rise to some neutron-rich isotopes.

To elucidate this, Table 2 lists the yields of ${ }^{56} \mathrm{Ni},{ }^{54} \mathrm{Fe}$ and ${ }^{58} \mathrm{Ni}$ from nucleosynthesis post-processing calculations for detonations of a system with $M_{\mathrm{WD}}=1.06 \mathrm{M}_{\odot}$ in which we adopted initial mass fractions of $0,2.5$ and 7.5 per cent for ${ }^{22} \mathrm{Ne}$ (corresponding to metallicities of $Z \sim 0,1$ and $\left.3 Z_{\odot}\right)$. As expected, in the absence of ${ }^{22} \mathrm{Ne}$, only a tiny mass of stable IGEs is produced $\left(\sim 10^{-3} \mathrm{M}_{\odot}\right)$. However, for $X\left({ }^{22} \mathrm{Ne}\right)=0.025$, the model predicts around $0.05 \mathrm{M}_{\odot}$ of ${ }^{54} \mathrm{Fe}+{ }^{58} \mathrm{Ni}$. This is still smaller than the stable IGE mass found in most delayed detonation models and roughly a factor of five less than in the widely-used W7 model (Nomoto, Thielemann, \& Yokoi 1984, Nomoto et al. 1997). Nevertheless, it demonstrates that sub-Chandrasekhar mass models can produce a nonnegligible mass of stable IGEs. Further study is warranted to investigate whether this could address the constraints associated with modeling the nebular spectra and we note that studies of galactic chemical evolution have suggested that the ${ }^{58} \mathrm{Ni}$-yield of standard SNe Ia models (e.g. the W7 yields of Nomoto et al. 1997) may be too high by a factor of a few (Kobayashi et al. 2006).

\section{Summary}

Detonation of sub-Chandrasekhar mass CO WDs can yield explosions that have the brightness and characteristic spectral features of SNe Ia. Since sub-Chandrasekhar mass WDs are relatively common, it is therefore worthwhile to investigate whether such explosions could be realized in nature. Detonation could be triggered following accretion from a He-rich companion or during a merger with a CO WD companion. Both of these models have several challenges to overcome but show sufficient promise to be worthy of ongoing study.

\section{Acknowledgements}

S.A.S thanks K. Nomoto and C. Kobayashi for several interesting and helpful discussions. This work was supported by the Deutsche Forschungsgemeinschaft via Emmy Noether Program (RO 3676/1-1), the Excellence Cluster EXC 153, and the Transregional Collaborative Research Center TRR 33. The work of F.K.R. is additionally supported by the ARCHES award 2011. We acknowledge that some of the results in this paper have been achieved using the PRACE Research Infrastructure resource JUGENE based in 
Germany at Forschungszentrum Jülich. In addition, computational resources for this project were provided by Forschungszentrum Jülich through the grants HMU13 and HMU14.

\section{References}

Arnett, W. D., Truran, J. W., \& Woosley, S. E., 1971, ApJ, 165, 87

Benetti, S., et al., 2005, ApJ, 623, 1011

Dan, M., Rosswog, S., Guillochon, J., \& Ramirez-Ruiz, E., 2011, ApJ, 737, 89

Fink, M., Röpke, F. K., Hillebrandt, W., Seitenzahl, I. R., Sim, S. A., \& Kromer, M., 2010, $A \& A, 514,53$

Guillochon, J., Dan, M., Ramirez-Ruiz, E., \& Rosswog, S., 2010, ApJ, 709, 64

Höflich, P., Khokhlov, A., Wheeler, C. J., Phillips, M. M., Suntzeff, N. B., \& Hamuy, M., 1996, ApJ, 472, L81

Kasen, D., Röpke, F. K., \& Woosley, S. E., 2009, Nature, 460, 869

Kobayashi, C., Umeda, H., Nomoto, K., Tominaga, N., \& Ohkubo, T., 2006, ApJ, 653, 1145

Krisciunas, K., Phillips, M. M., \& Suntzeff, N. B., 2004, ApJ, 602, L81

Kromer, M. \& Sim, S. A., 2009, MNRAS, 398, 1809

Kromer, M., Sim, S. A., Fink, M., Röpke, F. K., Seitenzahl, I.,R., \& Hillebrandt, W., 2010, ApJ, 719,1067

Livne, E. \& Arnett, D., 1995, ApJ, 452, 62

Mazzali, P. A., Röpke, F. K., Benetti, S., \& Hillebrandt, W., 2007, Science, 315, 825

Nomoto, K., Thielemann, F.-K., \& Yokoi, K., 1984, ApJ, 286, 644

Nomoto, K., Iwamoto, K., Nakasato, N., Thielemann, F.-K., Brachwitz, F., Tsujimoto, T., Kubo, Y., \& Kishimoto, N., 1997, Nuclear Physics A, 621, 467

Nugent, P., Phillips, M., Baron, E., Branch, D., \& Hauschildt, P., 1995, ApJ, 455, L147

Nugent, P., Baron, E., Branch, D., Fisher, A., \& Hauschildt, P. H., 1997, ApJ, 485, 812

Pakmor, R., Kromer, M., Röpke, F. K., Sim, S. A., Ruiter, A. J., \& Hillebrandt, W., 2010, Nature, 463,61

Pakmor, R., Hachinger, S., Röpke, F. K., \& Hillebrandt, W., 2011, A\& A, 528, 117

Pastorello, A., et al., 2007, MNRAS, 377, 1531

Pritchet, C. J. \& Howell, D. A., Sullivan M., 2008, ApJ, 683, 25

Ruiter, A. J., Belczynski, K., Sim, S. A., Hillebrandt, W., Fryer, C. L., Fink, M., \& Kromer, M., 2011, MNRAS, in press (arXiv:1011.1407)

Seitenzahl, I. R., Meakin, C. A., Townsley, D. M., Lamb, D. Q., \& Truran, J. W., 2009, ApJ, 696,515

Seitenzahl, I. R.; Ciaraldi-Schoolmann, F., \& Röpke, F. K., 2011, MNRAS, 414, 2709

Sim, S. A., 2007, MNRAS, 375, 154

Sim, S. A., Röpke, F. K., Hillebrandt, W., Kromer, M., Pakmor, R., Fink., M., Ruiter, A. J., \& Seitenzahl, I. R., 2010, ApJ, 714, L52

Stritzinger, M., Mazzali, P. A., Sollerman, J., \& Benetti, S., 2006, A $\&$ A, 460, 793

van Kerkwijk, M. H., Chang, P., \& Justham, S., 2010, ApJ, 722, L157

Woosley, S. E. \& Weaver, T. A., 1994, ApJ, 423, 371

Woosley, S. E. \& Kasen, D., 2011, ApJ, 734, 38 